\title{
Fair remuneration of energy consumption flexibility using Shapley value
}

\begin{abstract}
This paper proposes a new methodology for fair remuneration of consumers participation in demand response events. With the increasing penetration of renewable energy sources with a high variability; the flexibility from the consumers' side becomes a crucial asset in power and energy systems. However, determining how to effectively remunerate consumers flexibility in a fair way is a challenging task. Current models tend to apply over-simplistic and non-realistic approaches which do not incentivize the participation of the required players. This paper proposes a novel methodology to remunerate consumers flexibility, in a fair way. The proposed model considers different aggregators, which manage the demand response requests within their coalition. After player provide their flexibility, the remuneration is calculated based on the flexibility amount provided by the players, the previous participation in demand response programs, the localization of the players, the type of consumer, the effort put in the provided flexibility amount, and the contribution to the stability of the coalition structure using the Shapley value. Results show that by assigning different weights to the distinct factors that compose the calculation formulation, players remuneration can be adapted to the needs and goals of both the players and the aggregators.
\end{abstract}

Keywords: Demand Response, Fairness, Payoff allocation, Remuneration, Shapley Value.

\section{Introduction}

The increasing penetration of renewable energy sources is leading to major changes in power and energy systems all around the world [8]. The importance of consumers is increasing in this context, as they provide the potential to balance the variation of renewable-based generation through consumption flexibility.

Consumption flexibility may come from multiple sources, such as industry consumers, residential buildings, storage units, or electrical vehicles [18]. The different types of consumers or prosumers enable the system to reach consumption flexibility with different characteristics, e.g. in amount, location, duration and activation time; which makes the flexibility from different sources worth differently depending on the system needs.

The consumption flexibility may be activated by different means, usually associated to demand response programs or events [13]. Although some changes in current electricity market models are already taking place, market models are still not able to accommodate small-sized energy resources, and therefore, small consumers can only participate in flexibility transactions through aggregators, which guarantee the minimum volume to enable the market participation. 
While these aggregators are able to negotiate energy and/or flexibility, how they should distribute their incomes in order to effectively and fairly remunerate the consumers that are providing the flexibility is a challenging task. Although some studies are being made (e.g. [2]), the definition of remuneration models for the participation in demand response programs is not sufficiently explored, and solutions are yet not adequate.

Lessons in this field can be learnt from artificial intelligence applications which have solved similar problems. An example is in [1] with the application of the Shapley value to divide the profit of a marketers group among them in the scope of a cooperation model among agents in the electricity market. The Shapley value defines how important is each player to the overall cooperation, and what payoff can the player reasonably expect. the optimal cost-sharing rule that optimizes the price of anarchy, followed by the price of stability, is precisely the Shapley value cost-sharing rule. See [6] for a survey of the subject.

The Shapley value determines a payoff for each player based on the player's contribution to the stability of the coalition structure; e.g. in what amount is the player responsible for determining the coalition structure. This, however, does not directly represent the payoff in terms of utility that each player should get as outcome of the game. Considering the specific setup of the problem considered in this work, players payoff is received in the form of a remuneratory monetary compensation for the sale of their consumption flexibility. This is a direct result of players available amount of flexibility, among other considered parameters. Hence, the Shapley value cannot be used as a direct representation of the players payoff, but it can be considered as an influential factor in the payoff calculation, as means of rewarding players for their contribution to the stability of the coalitions structure. In this way, this paper proposes a remuneration definition methodology that distributes the total amount of revenue that the players are entitled to as a whole, among the involved players. This remuneration calculation formulation considers the monetary component that each player should receive from the sale of the flexibility amount, but also several other components that contribute to the quality of the provided service. In specific, the remuneration calculation considers: the flexibility amount, the previous participation in demand response programs, the localization of the player, the type of consumer (residential, industrial, etc.), the effort put in the provided flexibility amount, and the contribution to the stability of the coalition structure (Shapley value). Using the proposed remuneration calculation methodology, it is possible to remunerate the players involved in demand response events in a fair way, enabling the payment for their amount of provided flexibility, but also incentivizing important aspects for the system, such as the relevant type of consumer, the location in the network of the provided flexibility and the effort of the players, as well as their intrinsic contribution for the stability of the coalitions, which is essential for aggregators and operators to be able to define adequate programs directed to the specific groups.

After this introductory section, section 2 presents a discussion on the relevant work related to the present paper. Section 3 provides the formulation and description of the proposed methodology and section 4 presents the results achieved from the application of the proposed model. Finally, section 5 wraps up the paper with the main conclusions from the presented work. 


\section{Related work}

Developments in game theoretic models have potentiated their application to several research fields, including power and energy systems, see e.g. [17] for an overview on game theoretic methods in this domain. Worth highlighting is the work presented in [12], in which a game-theoretical model for energy scheduling of demand side resources is proposed. [3] presents an energy management model based on game theoretic assumptions, in [9] the optimization of the distribution system planning is performed using game theory, and in [15] a game theory-based strategy for electricity market participation is proposed. Several electricity market-driven simulation systems based on game theory concepts have also been introduced; e.g. [19] introduces a simulation system for energy storage devices management and operation. This problem is defined as a multi-player game, and Nash equilibrium is used to minimize the energy cost by reducing the peak demand. The Short-medium Run Electricity Market Simulator (SREMS) [11] is game theory-based and is able to support scenario analysis in the short-medium term and to evaluate market power.

One relevant, and often disregarded, aspect in this domain is the formation of coalitions between agents, so that they may improve their negotiation power and even for small players to gain access to market opportunities that are only accessible to large players. A recent and relevant review in coalition structure formation is provided in [16].

Consumer and demand response aggregation models in power and energy systems are typically based on clustering approaches. E.g. in [14] consumers are aggregated using an optimization-based clustering approach, as facilitator to their participation in electricity markets. The model proposed in [2] aggregates consumers demand response participation using hierarchical clustering and fuzzy C-means. Several remuneration schemes are also experimented by combining different groups of players and their minimum, average and maximum prices.

Remuneration models are also addressed in [4], which studies current remuneration models in electricity markets and demonstrated the need to extend the current electricity market design by additional remuneration mechanisms to reach imposed quotas of renewable generation and provide investment incentives for new firm capacity. The work presented in [21] considers the sensitivity of users to electricity prices to establish realtime pricing models considering price-based demand response measures by formulating a real-time pricing sale scheme. On the other hand, [7] proposes two formulations of a game-theoretic market equilibrium models for capacity markets with distinctive features. Moreover, the equilibrium models explicitly combine the capacity markets with markets for flexibility and indirect with remuneration for renewable energy sources.

A game-theoretical model is also applied in [1] to define a cooperation model among agents in the electricity market. The Shapley value is used to divide the profit of the marketers group among them. The Shapley value is also used in [10] to support the decision-making process of a peer-to-peer trading mechanism. The proposed game theoretic approach delivers distributed energy management solutions for individuals in the trading process considering both optimality and fairness among prosumers when trading energy among each other. In [20] the Shapley value helps to quantify the marginal 
contribution of each aggregator when dealing with incentives to electricity users to reduce demand in contingency situations.

The existing applications of the Shapley value in power and energy related problems suggest that this value may be suitable to address the problem discussed in this work. The calculation of the individual remuneration of consumers when participating in demand response events, through the distribution of the total revenue of the corresponding aggregator is presented in the following section.

\section{$3 \quad$ Proposed Methodology}

This section presents the proposed methodology for fair remuneration of flexibility provision, including the formulation used in this work. Fig. 1. presents the overview of the set up considered by the proposed work.

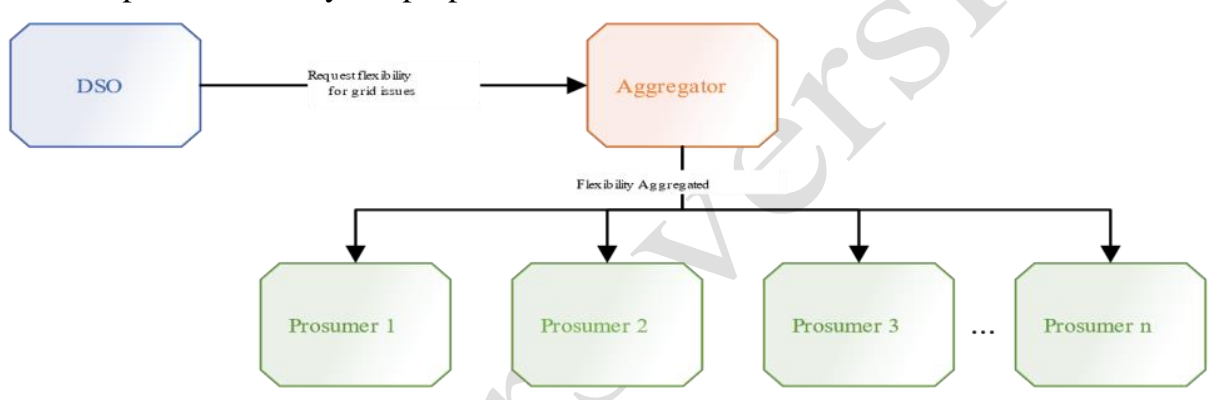

Fig. 1. Flexibility procurement scheme by DSO

As presented by Fig. 1, in cases of need from the system standpoint (e.g. contingency situations), the Distributed System Operator (DSO) requests consumption flexibility to one or several flexibility aggregators. The DSO will use this flexibility to solve issues in the distribution network, such as congestion management. This type of arrangement is proposed in a business model [5] of Dominoes project ${ }^{1}$. Each flexibility aggregator has a number of customers, which may be consumers or prosumers, and uses the flexibility from these customers in order to meet the requests from the DSO. After the costumers provide their flexibility, the DSO remunerates the aggregators for the provided flexibility, and the aggregators must share this income with the customers, in order to remunerate them for their services. Hence, Let $N$ be a finite, non-empty set of customers $\{1, \ldots, n\}$. Any subset $C$ of $N$ is called a coalition and is managed by an aggregator. The grand coalition is the set $N$ of all players. A coalition structure over $N$ is a collection of non-empty subsets $C S=\left\{C^{1}, \ldots, C^{k}\right\}$ such that

$$
\begin{aligned}
& \cup_{j=1}^{k} C^{j}=N \text { and } \\
& C^{i} \cap C^{j}=\emptyset \text { for any } i, j \in\{1, \ldots, k\} \text { such that } i \neq j .
\end{aligned}
$$

1 http://dominoesproject.eu/about/ 
The proposed remuneration definition methodology distributes the total amount of revenue among the involved players. This remuneration calculation formulation considers the monetary component that each player should receive from the sale of the flexibility amount, but also several other components that contribute to the quality of the provided service, namely: the flexibility amount, the previous participation in demand response programs, the localization of the player, the type of consumer (residential, industrial, etc.), the effort put in the provided flexibility amount, and the contribution to the stability of the coalition structure (Shapley value).

In equation (1), the aggregator renumeration is presented. In this case, it is considered that the DSO makes a request for flexibility to the aggregator. This request includes the price and amount of flexibility required by the DSO.

$$
R_{\text {agg }}=\text { Price }_{\text {agg }} \times A_{\text {agg }}
$$

where $R_{\text {agg }}$ represents the aggregator renumeration in $€$, Price $_{\text {agg }}$ represent the price in $€ / \mathrm{kW}$ that DSO will pay and $A_{\text {agg }}$ is the flexibility amount provided by the aggregator agg.

As shown by equation (2), for each aggregator, the sum of the amount of flexibility $A_{i}$, in kW, provided by each customer $i$ is equal to the total amount of flexibility provided by the aggregator to the DSO.

$$
A_{\text {agg }}=\sum_{i=1}^{N} A_{i}
$$

Equation (3) represents the price Price $_{i}$ that the aggregator will pay to each of its aggregates. The price represents $90 \%$ of the price the aggregator receives from the DSO, this represents the profit of $10 \%$ to the aggregator.

$$
\text { Price }_{i}=0,9 \times \text { Price }_{\text {agg }}
$$

Equation (4) represents $R_{i}^{1}$, which is in the basis remuneration of player $i$, and is calculated by multiplying the amount of flexibility provided by customer $i$ by the price payed by the aggregator to player $i$.

$$
R_{i}^{1}=\text { Price }_{i} \times A_{i}
$$

In (5) is defined the proposed remuneration model $R_{i}^{2}$.

$$
R_{i}^{2}=\text { Price }_{i} \times\left(A_{i} \times \frac{v_{i}^{1}}{\sum v^{1}}\right)
$$

The term $R_{i}^{2}$ represent the renumeration in $€$ for player $i$ and $v_{i}^{1}$ represents a factor which influences the remuneration of each player. This factor is calculated from the attributes of each player, as explained as follows. The term $\sum v$ correspond to the sum of all characteristic function values of all players.

$$
v_{i}^{1}=\sum_{j=1}^{N A} w_{j, i}^{1} \times A t_{i, j}^{1}
$$

where $w_{j, i}^{1}$ is the weight of attribute $j$ considering player $i, A t_{j, i}^{1}$ correspond to the value for attribute $j$ considering player $i$ and $N A$ correspond to the total number of attributes. 


$$
\begin{gathered}
A t^{1}=\{\text { participation, location, facility type } \\
\text { confort affect, Shapley value }\}
\end{gathered}
$$

The $v_{i}^{1}$ factor is calculated considering the attributes of each player that should influence the remuneration of the players, either by incentivizing certain characteristics or penalizing them. The considered attributes are (i) the previous participation in demand response programs, in order to benefit the players that contribute regularly to this type of programs, and thus incentivize players participation; (ii) the relevance of the location of each player, i.e. if the location of the customer is more or less beneficial to the system (if the reduction of consumption in the specific location contributes to effective power flow); (iii) the relevance of the type of facility, e.g. residential, commercial, industrial; (iv) the effort placed by the customer in the flexibility provision, measures by the relative amount of flexibility provides in relation to the total amount of consumption of the player, in order to reward players that make bigger efforts (provide a larger percentage of relative flexibility); and (v) the contrition of the players to the stability of the coalitions, measured by the Shapley value.

Equation (8) represents the preliminary characteristic function of player $i$, used to calculate the Shapley value, the term $w_{j, i}^{2}$ represent the weight for attributes considering all attributes in $A t^{2}$ set.

$$
v_{i}^{2}=\sum_{j=1}^{N A} w_{j, i}^{2} \times A t_{i, j}^{2}
$$

The $v_{i}^{2}$ factor is obtained considering the attributes of each player, except from the Shapley value. I.e. are considered the participation ratio, the location of each player, the type of facility and the comfort affect.

$$
A t^{2}=\{\text { participation, location, facility type, confort affect }\}
$$

where, $A t_{j, i}^{2}$ correspond to the value for attribute $j$ considering player $i$.

Equation (10) specifies the range of the attributes. All attributes are ranged in the interval $[0,1]$. The calculation of the relevance of the type of facility is performed according to equation (11)

$$
A t_{j, i}=\left\{\begin{array}{l}
{[0,1], \quad \text { if } j=1,2,4} \\
D_{c}, \quad \text { else if } j=3
\end{array}\right.
$$

The the relevance of the type of facility is calculated through the diversity $D_{C}$ among the players included in the same coalition $C$, considering their intrinsic characteristics. $D_{C}$ is calculated as in equation (11).

$$
\begin{gathered}
D_{C}=\frac{1}{G} \sum_{g=1}^{G} s \sigma_{g}^{C} \\
s \sigma_{g}^{C}=\frac{\sigma_{g}^{C}}{\left(\frac{g_{\max }-g_{\min }}{2}\right)}
\end{gathered}
$$




$$
\begin{gathered}
\sigma_{g}^{C}=\sqrt{\frac{1}{T_{C}} \sum_{t=1}^{T_{C}}\left(g_{t}-\mu_{g}^{C}\right)^{2}} \\
\mu_{g}^{C}=\frac{1}{T_{C}} \sum_{t=1}^{T_{C}} g_{t}
\end{gathered}
$$

where $G$ is the number of components that define the diversity of players in each coalition (e.g. volume of flexibility, type of consumer, price). Hence, $D_{C}$ calculates the average scaled standard deviation $s \sigma_{g}^{C}$ in coalition $C$ for all characteristics $g$ in a way that $s \sigma_{g}^{C} \in[0,1]$. For each characteristic $g, \sigma_{g}^{C}$ considers the values associated to each member $t$ of the coalition $C$. Firstly, the mean value $\mu_{g}^{C}$ for each characteristic $g$ in the coalition $C$ is calculated as in (14); then we reach the mean deviation $\sigma_{g}^{C}$ of each value of characteristic $g$ (from each player in coalition $C$ ) to the mean, as in (13). The standard deviation $\sigma_{g}^{C}$ is scaled according to the maximum and minimum values of $g$, in order to get $s \sigma_{g}^{C}$, as showed in (12). Finally, the average between the $s \sigma_{g}^{C}$ of all characteristics is calculated, giving us the value of $D_{C}$, as in (11).

The Shapley value is calculated according to equation (15). It is one way to distribute the total gains to the players, assuming that they all collaborate. It is a "fair" distribution in the sense that it is the only distribution with certain desirable properties listed below.

$$
\varphi_{i}\left(v^{2}\right)=\sum_{S \subseteq N \backslash\{i\}} \frac{|S| !(N-|S|-1) !}{N !}\left(v^{2}(S \cup\{i\})-v^{2}(S)\right)
$$

where $N$ is the total number of players and the sum extends over all subsets $S$ of $N$ not containing player $i$. The formula can be interpreted as follows: imagine the coalition being formed one actor at a time, with each actor demanding their contribution $v^{2}(S \cup\{i\})-v^{2}(S)$ as a fair compensation, and then for each actor take the average of this contribution over the possible different permutations in which the coalition can be formed.

\section{$4 \quad$ Numerical results}

\subsection{Specifications}

The setup of this case study is a set of 3 players, which are part of the same coalition. The aggregator in charge of managing this coalition receives a request from the DSO to provide an amount of flexibility in a certain period in time. Each of the players provides a specific volume of flexibility, and the aggregator uses the proposed approach to calculate the remuneration for each of the players.

Table 1 shows the attributes characterization for all players. The amount is the flexibility value that the player reports. The participation attribute is obtained from an analysis in the historical participation. Location value is obtained by the location of each 
player in the distribution network. In the facility type value are included the characteristic of each facility. The comfort affect value is related with quantity of load that each player is predisposed to cut, taking into account the total load that is consuming.

Table 1. Attributes characterization for the three considered players

\begin{tabular}{llllll}
\hline Players & Amount & Participation & Location & Facility type & Comfort affect \\
\hline A & 0 & 0,5 & 0,5 & 0,5 & 0 \\
B & 5 & 0,9 & 0,9 & 0,9 & 0,9 \\
C & 10 & 0,3 & 0,3 & 0,3 & 0,3 \\
\hline
\end{tabular}

From Table 1 one can see that player A does not provide any flexibility. Player B and player $\mathrm{C}$ provide 5 and $10 \mathrm{~kW}$ respectively. Player $\mathrm{C}$ has a larger amount to sell, but player $\mathrm{B}$ has better attributes values. It is expected that the renumeration has into account all attributes and thus fairly remunerate player $\mathrm{C}$, but also compensate player B for the good attributes.

Table 2 presents the values of input for equation (6) in term $w_{j, i}^{1}$. The table considers four different scenarios. In each scenario for each player the sum of all attribute weight must be equal to 1 . With the creation of scenarios, we try to study the influence of attributes weight in the final renumeration for each player. In specific, Scenario 1 provides a large weight to the amount, thus expecting to benefit players that provide larger volumes of flexibility regardless of their characteristics; Scenario 2 represents the opposite situation, by rewarding the other characteristics over the amount of flexibility; Scenario 3 considers a more balanced case; and Scenario 4 defines a higher weight for the stability of the coalitions over the remaining attributed.

Table 2. Weight specification for all attributes

\begin{tabular}{llllllll}
\hline $\begin{array}{l}\text { Sce- } \\
\text { nario }\end{array}$ & $\begin{array}{l}\text { Play- } \\
\text { ers }\end{array}$ & Amount & $\begin{array}{l}\text { Participa- } \\
\text { tion }\end{array}$ & $\begin{array}{l}\text { Loca- } \\
\text { tion }\end{array}$ & $\begin{array}{l}\text { Facility } \\
\text { type }\end{array}$ & $\begin{array}{l}\text { Comfort af- } \\
\text { fect }\end{array}$ & $\begin{array}{l}\text { Shap- } \\
\text { ley }\end{array}$ \\
\hline \multirow{4}{*}{1} & A & 0,75 & 0,05 & 0,05 & 0,05 & 0,05 & 0,05 \\
& B & 0,75 & 0,05 & 0,05 & 0,05 & 0,05 & 0,05 \\
& C & 0,75 & 0,05 & 0,05 & 0,05 & 0,05 & 0,05 \\
& A & 0,06 & 0,188 & 0,188 & 0,188 & 0,188 & 0,188 \\
& B & 0,06 & 0,188 & 0,188 & 0,188 & 0,188 & 0,188 \\
& C & 0,06 & 0,188 & 0,188 & 0,188 & 0,188 & 0,188 \\
& A & 0,3 & 0,14 & 0,14 & 0,14 & 0,14 & 0,14 \\
& B & 0,3 & 0,14 & 0,14 & 0,14 & 0,14 & 0,14 \\
& C & 0,3 & 0,14 & 0,14 & 0,14 & 0,14 & 0,14 \\
& A & 0,1 & 0,1 & 0,1 & 0,1 & 0,1 & 0,5 \\
4 & B & 0,1 & 0,1 & 0,1 & 0,1 & 0,1 & 0,5 \\
& C & 0,1 & 0,1 & 0,1 & 0,1 & 0,1 & 0,5 \\
\hline
\end{tabular}

For this case study, the price that the DSO pays to the aggregator for the flexibility is fixed in $0.04 € / \mathrm{kWh}$. With the application of equation (3) the price for each player is $0.036 € / \mathrm{kWh}$. The flexibility request from the DSO to the aggregator is set at $15 \mathrm{~kW}$. 


\subsection{Results}

Table 3 presents the value of the characteristic function for all possible coalitions. This function is needed for inputs in the calculation of Shapley value.

Table 3. Values of characteristic function

\begin{tabular}{llllllll}
\hline Scenario & $v^{1}(A)$ & $v^{1}(B)$ & $v^{1}(C)$ & $v^{1}(A B)$ & $v^{1}(A C)$ & $v^{1}(B C)$ & $v^{1}(A B C)$ \\
\hline 1 & 0 & 4,18 & 8,06 & 4,18 & 8,06 & 12,24 & 12,24 \\
2 & 0 & 1,146 & 0,882 & 1,146 & 0,882 & 2,028 & 2,028 \\
3 & 0 & 2,13 & 3,21 & 2,13 & 3,21 & 5,34 & 5,34 \\
4 & 0 & 2,13 & 3,21 & 2,13 & 3,21 & 5,34 & 5,34
\end{tabular}

The values of characteristics function are obtained from application of equation (8). The $w_{j, i}^{2}$ are based on values of Table 2 but the weight of Shapley value is removed, and the other weight is normalized in a scale of 0 to 1 . In Table 4 are present the values of Shapley values obtained from the application of equation (15).

Table 4. Shapley values

\begin{tabular}{llll}
\hline Scenario & Players & Shapley value & Relative Shapley value \\
\hline \multirow{3}{*}{1} & A & 1,293 & 0,106 \\
& B & 5,473 & 0,447 \\
& C & 5,473 & 0,447 \\
2 & A & $-0,088$ & 0 \\
& B & 1,058 & 0,500 \\
& C & 1,058 & 0,500 \\
3 & A & 0,360 & 0,067 \\
& B & 2,490 & 0,466 \\
& C & 2,490 & 0,466 \\
4 & A & 0,360 & 0,067 \\
& B & 2,490 & 0,466 \\
& C & 2,490 & 0,466
\end{tabular}

In Table 4 are present the results of Shapley value and relative Shapley value. The relative Shapley value is a normalization of Shapley value in $[0,1]$. Analyzing Table 4, players B and C have, in all scenarios the same Shapley value; meaning that these players' contribution to the stability of the coalition process is the same.

Table 5 presents the values for the different renumerations approach, namely the basis remuneration, as in equation (4), in which the amount of flexibility is multiplied by the price; the proposed approach without considering the Shapley value, which is obtained using equation (5) but the term $v_{i}^{1}$ come from equation (8). The Shapley value renumeration is obtained using the relative Shapley value present in Table 4 multiplied with the price for flexibility present in equation (3). The proposed approach with Shapley value renumeration is obtained using equation (5) and the values for $v_{i}^{1}$ are obtained in equation (6). 
Table 5. Results for the different renumerations approaches

\begin{tabular}{llllll}
\hline \multirow{2}{*}{$\begin{array}{l}\text { Scenario } \\
1\end{array}$} & Players & Basis & $\begin{array}{l}\text { Proposed approach } \\
\text { without Shapley value }\end{array}$ & $\begin{array}{l}\text { Shapley } \\
\text { value }\end{array}$ & $\begin{array}{l}\text { Proposed approach } \\
\text { with Shapley value }\end{array}$ \\
\hline & A & 0 & 0 & 0,057 & 0 \\
& B & 0,180 & 0,184 & 0,241 & 0,185 \\
& C & 0,360 & 0,356 & 0,241 & 0,355 \\
2 & A & 0 & 0 & 0 & 0 \\
& B & 0,180 & 0,305 & 0,270 & 0,291 \\
& C & 0,360 & 0,235 & 0,270 & 0,249 \\
& A & 0 & 0 & 0,036 & 0 \\
& B & 0,180 & 0,215 & 0,252 & 0,211 \\
& C & 0,360 & 0,325 & 0,252 & 0,329 \\
& A & 0 & 0 & 0,036 & 0 \\
& B & 0,180 & 0,215 & 0,252 & 0,241 \\
& C & 0,360 & 0,325 & 0,252 & 0,299 \\
\hline
\end{tabular}

Fig. 2 presents the values of Table 5 in order to facilitate the interpretation of the different forms of renumeration.

In Fig. 2 are present the four different types of renumeration. The four plots have in vertical axes the values of profit and in horizontal axes the different scenarios $(1,2,3$ and 4), and for each scenario the respective three players. From Fig 2 a) it is visible that the basis remuneration is the same regardless of the scenario, with player $\mathrm{C}$ receiving twice the payoff of player B, since it provides twice the flexibility amount; and player A receiving nothing. Fig 2 b) shows that in Scenario 1, which defines a very large weight to the amount of flexibility, results are similar to the basis remuneration, similarly to Fig 2. d). In Scenario 2, which defines a smaller weight for the flexibility amount against the other characteristics, player $\mathrm{C}$ is benefited, and has even a larger remuneration than player $\mathrm{B}$ even though it provides a smaller amount of flexibility. In Scenario 3, which considers a more balanced weight distribution, the remuneration distribution is more balanced as well between players $\mathrm{B}$ and $\mathrm{C}$, with $\mathrm{C}$ being attributed a higher remuneration for its larger amount of flexibility, but player B being rewarded by its good characteristics. Finally, in Scenario 4, considering the Shapley value with larger weight; the remuneration values of both players are more balanced, since their Shapley value is the same, hence, since both contribute the same to the stability of the coalitions, they end up being more balanced.

\section{Conclusions}

This paper has proposed a methodology to enable a fair renumeration model. The proposed renumeration model considers different player attributes, the participation ratio, the location of each player, the type of facility, the comfort affect and the value of Shapley. Results show that the remuneration can be defined taking into account the attributes of the players and their actual contribution in terms of flexibility amount and 
stability. It is seen that considering only the Shapley values is not adequate to define a fair remuneration model, but considering the Shapley values as part of the remuneration model benefits the remuneration process. For future work it is suggested to explore this proposed methodology with the interaction between a greater number of players, considering also the physical limitations that the distribution network may present.

a)

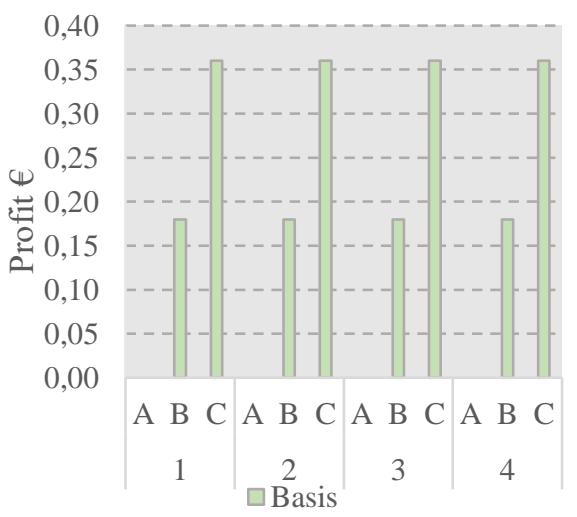

c)

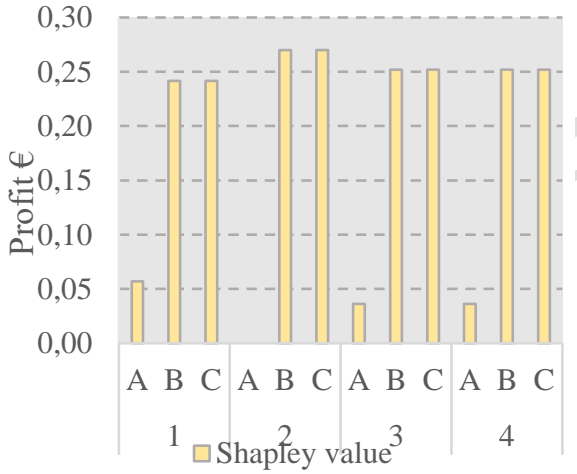

b)

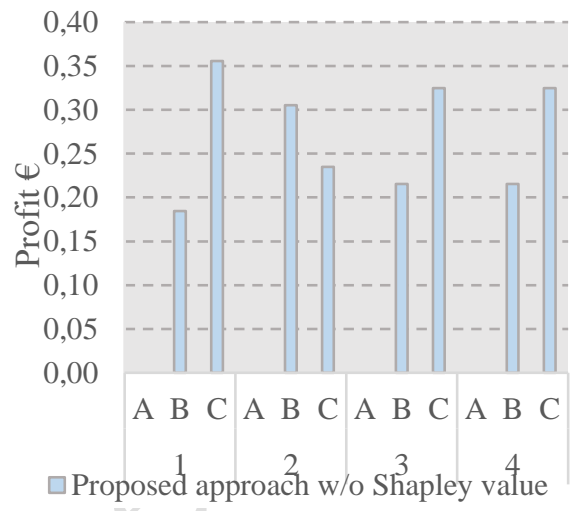

d)

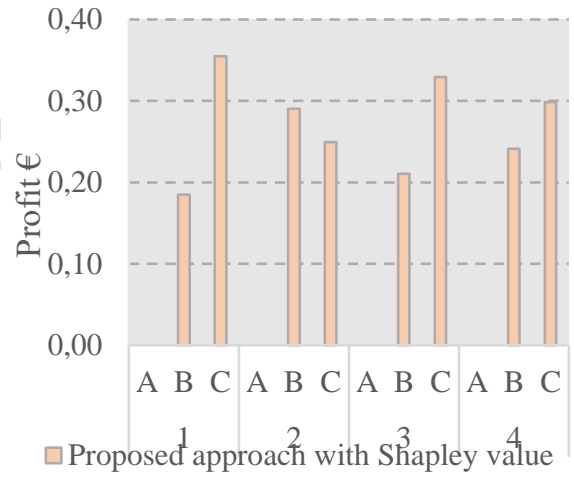

Fig. 2. Different renumeration approaches, a) Basis renumeration, b) Proposed approach without Shapley value, c) Shapley value renumeration and d) Proposed approach with Shapley value

\section{References}

1. Acuña, L.G. et al.: Cooperation model in the electricity energy market using bi-level optimization and Shapley value. Oper. Res. Perspect. 5, 161-168 (2018).

2. Faria, P. et al.: Aggregation and Remuneration of Electricity Consumers and Producers for the Definition of Demand Response Programs. IEEE Trans. Ind. Informatics. PP, 99, 1-1 (2016).

3. Gao, B. et al.: Game-Theoretic Energy Management for Residential Users with Dischargeable Plug-in Electric Vehicles, (2014). https://doi.org/10.3390/en7117499.

4. Gerres, T. et al.: Rethinking the electricity market design: Remuneration mechanisms to 
reach high RES shares. Results from a Spanish case study. Energy Policy. 129, 13201330 (2019).

5. Guimarães, A. et al.: Dominoes Project Deliverable 5.1 - Formulation of alternative local market place enabled business models.

6. Hart, S.: Shapley Value BT - Game Theory. Presented at the (1989).

7. Höschle, H. et al.: Electricity markets for energy, flexibility and availability - Impact of capacity mechanisms on the remuneration of generation technologies. Energy Econ. 66, 372-383 (2017).

8. Hossain, M.S. et al.: Role of smart grid in renewable energy: An overview. Renew. Sustain. Energy Rev. 60, 1168-1184 (2016)..

9. Li, R. et al.: Game Optimization Theory and Application in Distribution System Expansion Planning, Including Distributed Generation, (2013).

10. Long, C. et al.: A game theoretic approach for peer to peer energy trading. Energy Procedia. 159, 454-459 (2019).

11. Migliavacca, G.: SREMS: A short-medium run electricity market simulator based on game theory and incorporating network constraints. In: 2007 IEEE Lausanne POWERTECH, Proceedings. pp. 813-818 (2007).

12. Mohsenian-Rad, A.H. et al.: Autonomous demand-side management based on gametheoretic energy consumption scheduling for the future smart grid. IEEE Trans. Smart Grid. 1, 3, 320-331 (2010).

13. Müller, T., Möst, D.: Demand Response Potential: Available when Needed? Energy Policy. 115, 181-198 (2018).

14. Parvania, M. et al.: Optimal Demand Response Aggregation in Wholesale Electricity Markets. IEEE Trans. Smart Grid. 4, 4, 1957-1965 (2013).

15. Pinto, T. et al.: Strategic bidding in electricity markets: An agent-based simulator with game theory for scenario analysis. Integr. Comput. Aided. Eng. 20, 4, 335-346 (2013).

16. Rahwan, T. et al.: Coalition structure generation: A survey. Artif. Intell. 229, 139-174 (2015). https://doi.org/https://doi.org/10.1016/j.artint.2015.08.004.

17. Saad, W. et al.: Game-Theoretic Methods for the Smart Grid: An Overview of Microgrid Systems, Demand-Side Management, and Smart Grid Communications. IEEE Signal Process. Mag. 29, 5, 86-105 (2012).

18. Tan, K.M. et al.: Integration of electric vehicles in smart grid: A review on vehicle to grid technologies and optimization techniques, (2016).

19. Vytelingum, P. et al.: Agent-based micro-storage management for the smart grid. In: Proceedings of the International Joint Conference on Autonomous Agents and Multiagent Systems, AAMAS. pp. 39-46 (2010).

20. Wang, J. et al.: Ensuring profitability of retailers via Shapley Value based demand response. Int. J. Electr. Power Energy Syst. 108, 72-85 (2019).

21. Zhang, P. et al.: Analysis of Power Sales Strategies Considering Price-Based Demand Response. Energy Procedia. 158, 6701-6706 (2019).. 